\title{
UNMANNED AERIAL SYSTEMS IN THE PROCESS OF JURIDICAL VERIFICATION OF CADASTRAL BORDER
}

\author{
M. Rijsdijk ${ }^{\mathrm{a}}$, W.H.M. van Hinsbergh ${ }^{\mathrm{a}}$, W. Witteveen ${ }^{\mathrm{a}}$ G.H.M. ten Buuren ${ }^{\mathrm{b}}$, G.A. Schakelaar ${ }^{\mathrm{b}}$, G. Poppinga ${ }^{\mathrm{c}}$, M. van \\ Persie $^{c}$, R. Ladiges ${ }^{c}$ \\ ${ }^{a}$ Kadaster, Hofstraat 110, 7311 KZ, Apeldoorn, The Netherlands - martijn.rijsdijk@kadaster.nl \\ ${ }^{\mathrm{b}}$ National Unit of the Netherlands Police, Hoofdstraat 54, 3972LB Driebergen, The Netherlands - \\ gerard.ten.buuren@klpd.politie.nl \\ ${ }^{\mathrm{c}}$ NLR, A. Fokkerweg 2, 1059CM Amsterdam, The Netherlands - Mark.van.Persie@nlr.nl
}

KEY WORDS: UAS, Surveying, High Resolution, SFM, ortho-photo, cadaster, juridical verification of borders

\begin{abstract}
:
Quite often in the verification of cadastral borders, owners of the parcels involved are not able to make their attendance at the appointed moment in time. New appointments have to be made in order to complete the verification process, and as a result often costs and throughput times grow beyond what is considered to be acceptable. To improve the efficiency of the verification process an experiment was set up that refrains from the conventional terrestrial methods for border verification. The central research question was formulated as "How useful are Unmanned Aerial Systems in the juridical verification process of cadastral borders of ownership at het Kadaster in the Netherlands?"

For the experiment, operational evaluations were executed at two different locations. The first operational evaluation took place at the Pyramid of Austerlitz, a flat area with a 30m high pyramid built by troops of Napoleon, with low civilian attendance. Two subsequent evaluations were situated in a small neighbourhood in the city of Nunspeet, where the cadastral situation recently changed, resulting from twenty new houses that were build. Initially a mini-UAS of the KLPD was used to collect photo datasets with less than $1 \mathrm{~cm}$ spatial resolution. In a later stage the commercial service provider Orbit Gis was hired. During the experiment four different software packages were used for processing the photo datasets into accurate geo-referenced ortho-mosaics. . In this article more details will be described on the experiments carried out. Attention will be paid to the mini-UAS platforms (AscTec Falcon 8, Microdrone MD-4), the cameras used, the photo collection plan, the usage of ground control markers and the calibration of the camera's. Furthermore the results and experiences of the different used SFM software packages (Visual SFM/Bundler, PhotoScan, PhotoModeler and the Orbit software) will be shared.
\end{abstract}

\section{INTRODUCTION}

In the Netherlands Kadaster is the national land registration service and mapping agency. Kadaster is responsible for maintaining an actual and complete registration of deeds of ownership, cadastral maps and the juridical borders of real estate, see also Figure-1. Kadaster needs to inform those who are part of the transactions about the current situation.

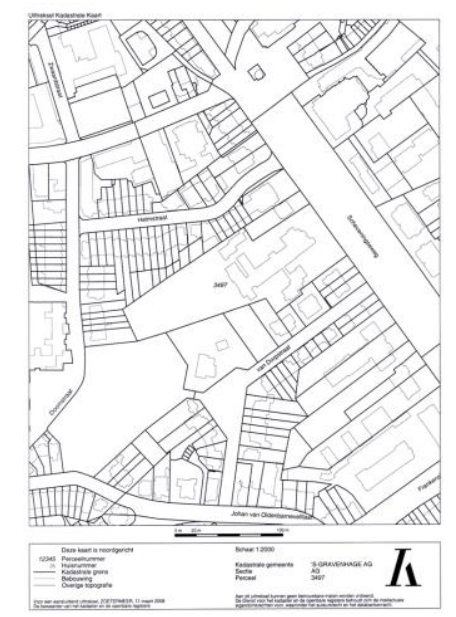

Figure 1: Cadastral map with verified borders
When ownership of one or more parts of a parcel changes, seller(s) and buyer(s) are legally obliged to identify the new boundaries for the cadastral employee. After verification, the new cadastral situation is created through surveying and cartographical activities. As a result the new situation will be represented in the cadastral registration. However, often the involved stakeholders are not able to appear during these appointments at the last moment when the cadastral employer is often already at the location. Because the presence of the owner is necessary, the verification process stocks and a new appointment has to be made. In the meantime, the cadastral map is no longer actual.

Kadaster currently seeks ways to improve their production process and make it more efficient. This could result in lower tariffs for customers and a higher service level [Oosterbroek, 2012]. Important criteria that would make the process more efficient are 1) customers should not have make their attendance during daytime 2) Owners still need to be able to do the verification of the new cadastral situation and 3) People need to be able to actually see a "real" insight in the new cadastral situation for a right verification.

This insight could be achieved by using high-resolution aerial photographs. Those images give a truly view of the actual topographical situation, which would eliminate the necessity for all stakeholders - seller(s), buyer(s) and cadastral officer - 
to be present on site simultaneously. Instead, the seller(s) and buyer(s) would be able to identify the new boundaries on the image, possibly at the moment of transaction in the notary office. The cadastral officer would then be able to sketch the boundary on a soft or hard copy of the aerial image.

But to use these photographs for verification, it should be possible to determine the new situation with a geometric accuracy that is comparable with conventional land surveying methods. These terrestrial methods have a geometrical precision of 6 centimetres or less [HTW, 1994]. Therefore, accurate geometrically corrected ortho-mosaics need to be created from the high-resolution aerial images [Cunningham, 2011]. An ortho-mosaic gives an equal and uniform view of the local terrain situation with a uniform scale and minimal geometric distortions, so that true coordinates and distances can be measured [Flury, 2011].

Using ortho-mosaics as created from conventional aerial images are unsuitable, because of the relatively high altitude and related low spatial resolution and its costs. Images captured by Unmanned Aerial Systems (UAS) seem to have appropriate characteristics: flying at a relatively low altitude (40m) results in high spatial resolutions and for covering small areas the operation costs and relatively low and the flexibility is relatively high. From literature it was found that the obtained precision can be in the order of $3 \mathrm{~cm}$ [Manyoky, 2011], which would be enough to apply UAS imagery for boundary identification.

\section{EXPERIMENTS}

During spring 2011 the Product Innovation Department of Kadaster decided to seriously investigate the suitability of aerial images captured by UAS for the identification of property boundaries by executing a practical evaluation. As Kadaster had no previous experience with UAS and accurate Structure From Motion based photogrammetric processing techniques, an active cooperation was sought with the Dutch National Aerospace laboratory NLR. Jointly a plan for an operational test and evaluation of the technology and for experimenting with a number of relevant processing techniques was drafted. As NLR actively cooperates with the UAS departments of the National Unit of the Netherlands Police and the subject of research shows significant overlap with the area of detailed crime scene registration, the Netherlands Police responded positively on a request to join the experiments. The combined expertise and experience of the three parties provided an environment for rapid progress.

The central research question of the overall cooperation was formulated as "How useful are Unmanned Aerial Systems in the juridical verification process of cadastral borders of ownership at het Kadaster in the Netherlands?"

In total three experiments were conducted at two locations: Austerlitz and the city of Nunspeet.

\subsection{First experiment}

The first experiment was carried out 18 January 2012, at the Pyramid of Austerlitz, a flat sandy area surrounded by forest with a $30 \mathrm{~m}$ high pyramid, built as victory monument by troops of Napoleon, see Figure-2. This concerned a learning phase aimed at getting acquainted with the technology.

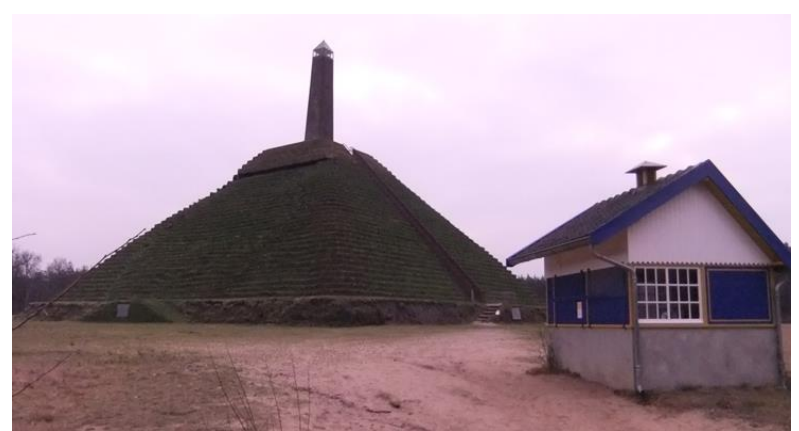

Figure 2: Pyramid of Austerlitz, January 2012.

At 08:00h first preparations at the location started, with placing 24 ground markers followed by accurate GNSS surveying, see Figure-3. Second, test flights were made with the AscTec Falcon UAS of the police to determine the optimal camera settings and grid for the mission plan. The area was split up in five parts to be covered in five separate flights. Around 13:00h, the measuring flights were started. The temperature was around freezing, which reduced the capacity of the batteries. The UAS flight duration was limited to less than 10 minutes instead of the nominal 20 minutes, after which the batteries needed to be recharged. Unfortunately, around $15 \mathrm{~h}$ PM it started to rain. At 15:00h, rainfall prompted cancellation of the last of five scheduled flights. A week later this fifth flight was still carried out.

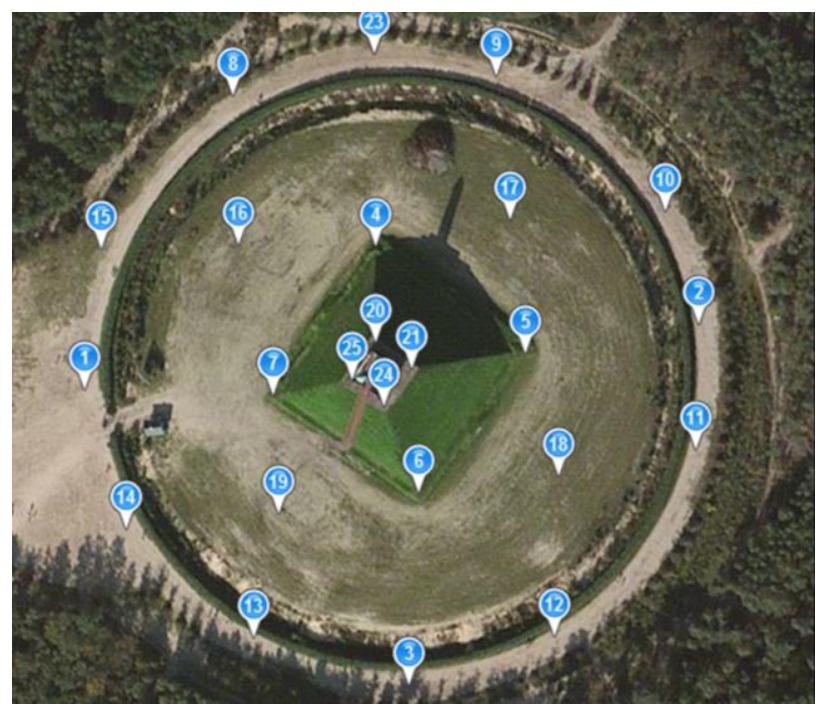

Figure-3: Overview of Austerlitz test area with locations of the ground control points

The flights resulted in a relevant dataset of 480 photo's with a resolution of $1 \mathrm{~cm}$ and an overlap of $80 \%$. An inventory was made of possible processing tools and processing activities were carried out with VisualSFM and PhotoModeler Scanner. The activities included bundle block adjustment and point cloud generation. Computer memory had to be extended and experience with different parameter settings was gained. First conclusions were that the obtained average geometrical accuracy was less than 10 centimetres, see also Figure-4. Although this was below the target precision of 6 centimetres, the insight obtained with this experiment and the room for enhancements made everyone enthusiast. 


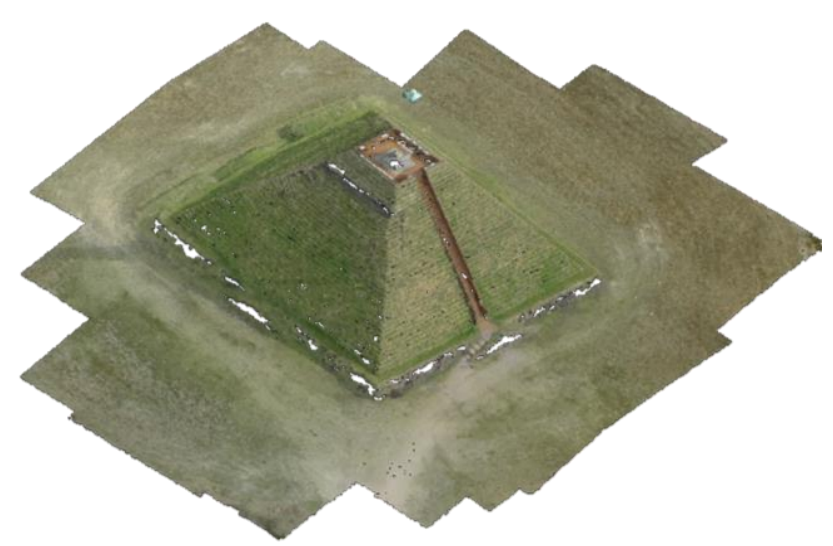

Figure-4: Computed 3D pointcloud of the Pyramid

\subsection{Second experiment}

The second experiment was conducted in Nunspeet on 29 March 2012. It concerned a real cadastral situation in which the parcel boundaries of 20 new houses had been identified and measured, see Figure-5.

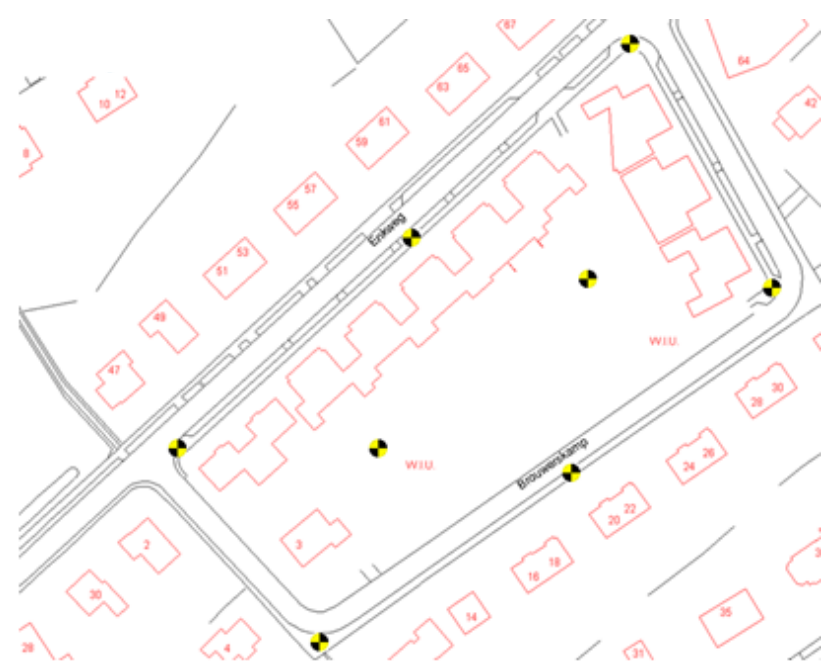

Figure-5: Overview of Nunspeet test area with locations of the ground control points

For the set-up of the experiment the gained experience from the first experiment was used. At 09:00h a start was made with the signalling and GNSS measuring of ground control points, now using professional markers. Besides also check points were measured at several characteristic places of the parcel borders. Also for this flight the AscTec Falcon UAS of the police was used. In the preparation phase municipal permission was arranged since the flights would be conducted over houses and civilians. A flight plan was prepared for the target areas, consisting of three sub-flights. First a test flight was made at $10.00 \mathrm{~h}$ to check the proper functioning of the system and the camera settings. Unfortunately during the third measuring flight a sudden wind blast threw the UAS off balance and the experiment had to be terminated at 11:30h. As a consequence the dataset was not fully complete. With the data of the two measuring flights and test flight a total of 105 photographs were obtained, covering $70 \%$ of the area with $1 \mathrm{~cm}$ detail and $80 \%$ overlap. After the flights also a set of calibration photographs was gathered. For the processing of this dataset use was made of the processing tools PhotoModeler Scanner and PhotoScan Professional. Especially with the last package the full processing chain could be handled, from alignment, point cloud generation, geo-referencing, mesh generation with texturizing, and ortho-photo generation. The resulting geometric accuracy of the ortho-photo was in the order of 5 centimetres.

In the execution of the operational tests and the processing of the resulting data, a thorough understanding of the relevant aspects and requirements on both the UAS operations and the processing software was formed. Insight also was obtained in the required regulations for operating a UAS, asking for permission by the regional government had to be arranged by Kadaster because of flying above houses and civilians. Also the use and placement of ground markers was improved; using more visible markers and putting these at better geometrical locations.

\subsection{Third experiment}

The third experiment involved the same test site in Nunspeet, but now executed by a commercial service provider. Orbit GIS, a commercial service provider for UAS based aerial photography based services, was hired to generate a set of aerial images and provide a resulting ortho-photo. This provided a reference for comparison of the experimental activities with commercially available services. Kadaster took care for the preparation of the flight plan and surveying's of ground markers. Because of the experiences with the previous two flights Kadaster was able to define the right specifications correctly and clear. On 19 June 2012 Orbit GIS finished their two shifts in Nunspeet in fifty minutes successfully with a correct dataset. The used UAS was a Microdrone MD-4 1000 Beta with an Olympus E-P3 OGT camera. The UAS flew on a altitude of 50 meters and an overlap of $80 \%, 360$ images were collected.

Orbit GIS also took care of the processing with an ortho-photo as result. Post-processing was started by importing the gathered data in Orbit software. Operators started with automatic step- and model creation, followed by auto-detection of points and matching images with the so called "Von Gruber" matching method. After Bundle Block Adjustment (BBA) the images were stitched geometrically and oriented correctly to each other. A Digital Terrain Model could be build and based on this an ortho-photo was created, see also Figure6. The average geometrical accuracy of all photos was 3 centimetres maximum! Because of the overlap of $80 \%$ it is also possible to create stereo-photos; on a screen it is possible to show the pictures in $3 \mathrm{D}$.

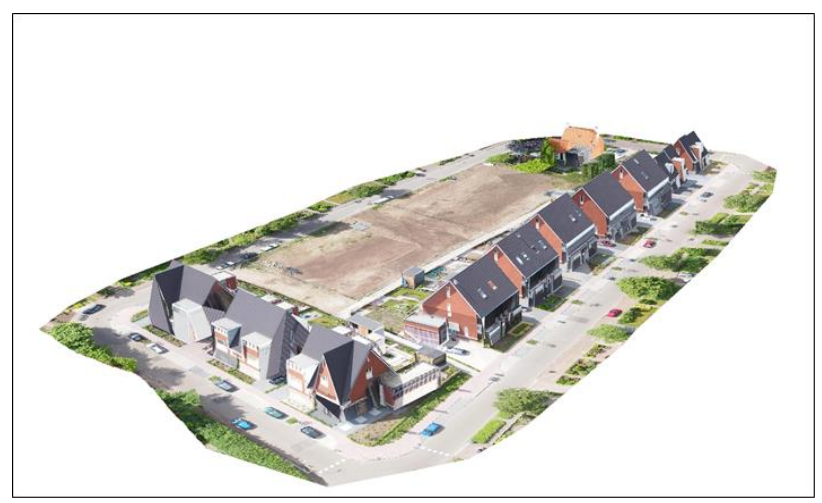

Figure 6: Textured 3D model created with elevation model and measured roof lines 


\section{TECHNICAL ASPECTS}

\subsection{Platforms and mission planning}

The UAS that were used were a Falcon 8 from Ascending Technologies (AscTec) in the first and second experiments, and a Microdone MD-4 1000 Beta in the third experiment (Figure 7).

The Falcon 8 is an octocopter (eight rotors), remains stable up to wind speeds of $10 \mathrm{~m} / \mathrm{sec}$ ( 5 Beaufort; fresh breeze), can carry a payload of $500 \mathrm{~g}$ and can stay in air for 20 minutes. Navigation and positioning is done using GNSS, an inertial measurement unit (IMU), a barometric height sensor and a compass. Usage was made of new functionality of the mission planning software to autonomously gather a block of photographs for an area based on a defined region of interest, overlap and altitude.

The Microdrone MD-4 1000 Beta is a quadrocopter (four rotors), can carry a payload of $1,200 \mathrm{~g}$ and can stay in the air for up to 45 minutes. Navigation and positioning is done using GNSS.

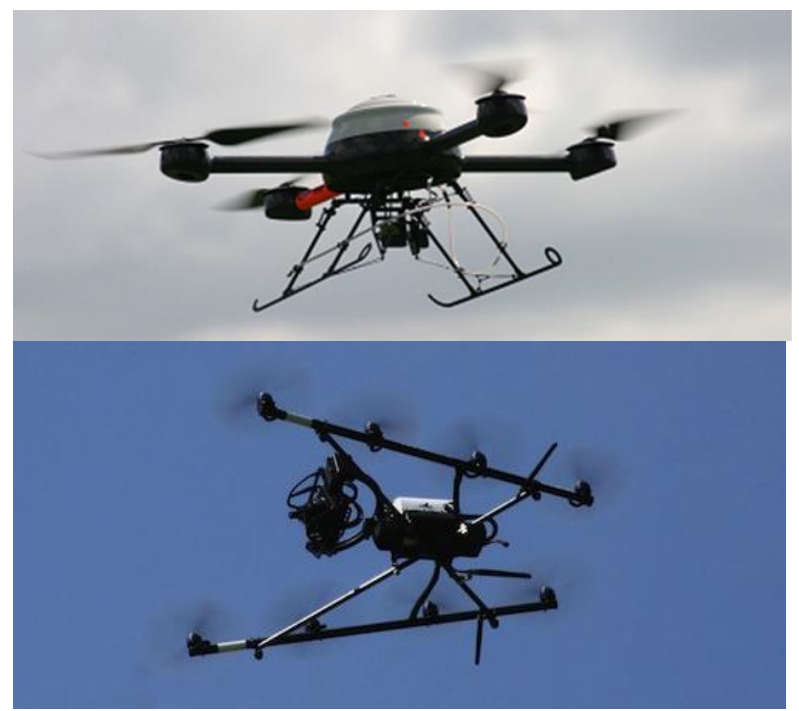

Figure 7: The Microdone MD-4 1000 (up) and AscTec Falcon 8 (down)

Both for the Falcon and Microdone special mission planning software was available that could generate a mission plan for autonomous flying a grid of positions where photographs are captured, such that a specified area of interest is covered with photos with required overlap and resolution, see also Figure-8. Based on the test fights for the Falcon it was specified to firstly wait some second at each position before taking the photo, so that maximal stability of the system was obtained. An important aspect also was to take a wide enough zone around the area of interest, so that the full area was covered with photos with enough overlap and also control points on the edges of the area are captured in enough photos.

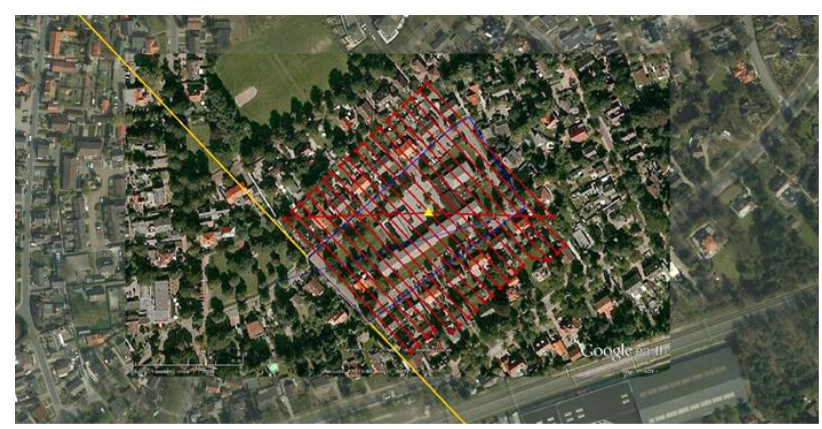

Figure 8: Flightplan second flight Nunspeet by Orbit GIS

\subsection{Cameras and calibration}

The camera on board of the Falcon was a Panasonic LX-3, which is a good quality compact camera with a focal length of $5.1 \mathrm{~mm}$ and has an image size of 3,648 x 2,736 pixels and a pixel size of $2 \mu \mathrm{m}$.

The camera on board of the Microdrone was an Olympus E-P3 OGT with a focal length of $17 \mathrm{~mm}$, an image size of $4,032 \mathrm{x}$ 3,024 pixels and a pixel size of $4.4 \mu \mathrm{m}$.

For the Panasonic camera the zoom lens was set to minimal zoom, the autofocus and built-in stabilization were disabled in order to have maximal geometric and radiometric stability in the photoset. A short shutter time of at least $1 / 1000 \mathrm{sec}$ was required to prevent blurring due to movements.

During the second experiment special attention was paid to the calibration of the Panasonic LX-3 camera. After the flight photographs were taken from a number of paper sheets with special printed targets, see also figure-9. With the PhotoModeler Scanner software automatic self-calibration of the camera could be done.

The camera calibration parameters were used in the 3D reconstruction software. For PhotoModeler Scanner this resulted in enhanced accuracy. For PhotoScan Professional it was found that more accurate results were obtained when the camera calibration values were computed by the software during the alignment procedure.

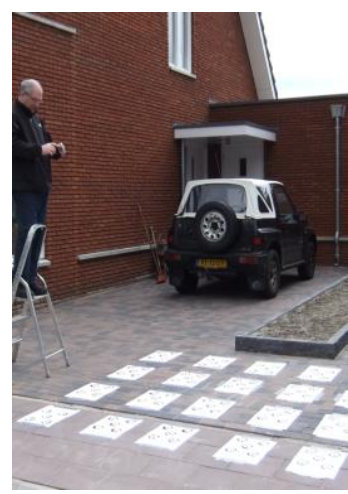

Figure 9: Procedure of the calibration of the camera

\subsection{Ground control}

During all experiments ground markers were placed at different locations in the area of interest and measured with the surveying method GNSS with accuracy in the order of 1 centimetre. In Austerlitz experiment usage was made of white CD's as markers. In the Nunspeet experiment more professional ground markers were used with a yellow/black 
cross and fit on metal nails for fixing, see also figure-10. In the third experiment also better locations were chosen for the control points, more to the borders and better spread over the area.

For the Nunspeet experiments additional GNSS measurements were made to determine the positions of the new parcel borders, involving fences, pavement and other characteristic elements.

Thirdly, the used UAS delivered the GPS positions of the camera at the moment that a picture was taken. These could be made available for the processing software.

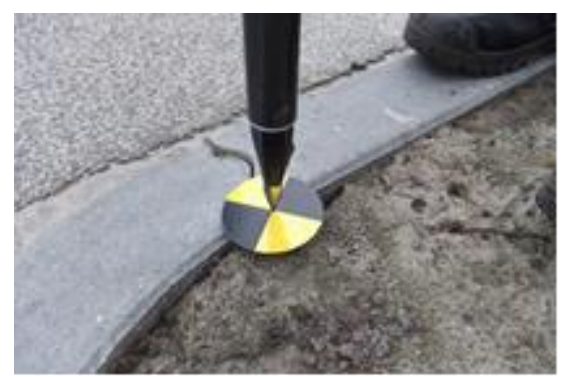

Figure 10: Professional ground marker for geometrical corrections

\subsection{Data processing}

For the processing of the acquired photographs datasets into accurate geo-referenced ortho-mosaics several Structure From Motion based software packages were used during the experiments: Bundler/PMVS2/Visual-SFM, PhotoScanProfessional, PhotoModeler Scanner and the Orbit software. Visual SFM [Visual SFM] is open software that builds upon the PhotoTourism software and adds a userfriendly GUI, GPU and multicore processing in feature detection, feature matching, and bundle adjustment.

PhotoScan-Professional [PhotoScan], PhotoModeler Scanner [PhotoModeler] are more operational commercial software package that more completely support the whole processing chain, including camera calibration, geo-referencing, generation of products and display of results.

The Orbit Strabo Photogrammetry software [Orbit] is digital photogrammetry suite, embedded in a corporate GIS environment as extension to Orbit GIS. It compiles bundle block adjustment, stereo viewing, DEM-generation and orthorectification. This software was operated by Orbit GT and only the results were evaluated by the project team.

Different functional elements are required for the generation of an ortho-mosaic: importing of photos and optionally accompanying position/attitude/camera parameters, photo alignment or bundle block adjustment, dense point cloud generation, geo-referencing with ground control points, orthomosaic generation. Additional functions may be $3 \mathrm{D}$ model computation (mesh generation texturizing), stereo display, coordinate measurement and integration with GIS information. The Visual SFM software is limited to photo alignment and point cloud generation. For geo-referencing and ortho-mosaic generation the result needs to be transferred to other software, which might not be straight forward.

Important is that not all packages involve all the steps that are required to obtain a final ortho-rectified product. PhotoScanProfessional has integrated all required functional steps in a user friendly way and can produce good quality ortho-mosaics or 3D models in most cases. The number of settings that can be manipulated is limited however, which means that for special situations the user has limited possibilities to manipulate the processing. PhotoModeler Scanner gives the user many more manipulation options, this at the cost of much more complicated software.

In general relatively powerful PC's are required with enough internal memory to handle datasets with hundreds of photos. Multiple cores reduce the processing time.

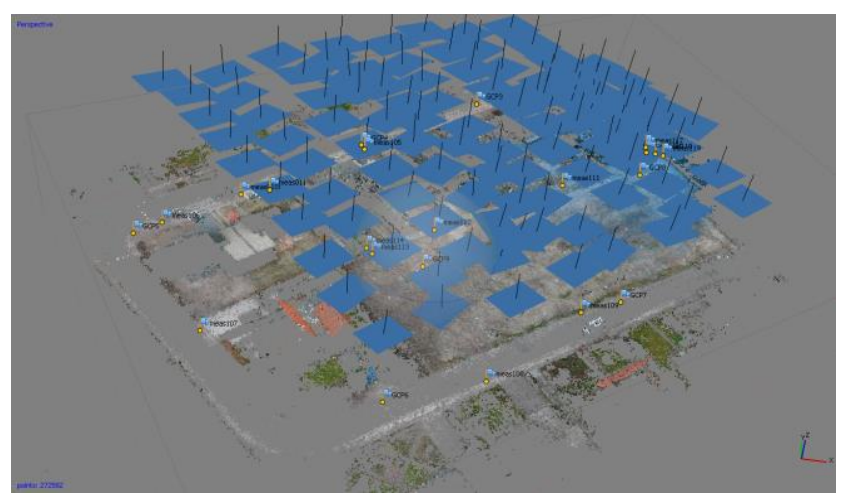

Figure-11: Result of bundle block adjustment by PhotoScan Professional for Nunspeet area.

The dense 3D model computation does not give optimal results in all cases. Especially vertical structures like building walls and fences give problems for the software. The orthogonal photos contain too little information on these facades, resulting in large deviations. Also the repetitive structure of the new roof tiles gave problems for the correlation process, see figure- 12 .

Unless these distortions in the $3 \mathrm{D}$ model, a relatively good ortho-mosaic could be produces in the case of PhotoScan Professional. This, because the errors mainly manifest in the Zdirection, which is transferred to the ground level.

The best quality ortho-mosaic is obtained by Orbit GIS, because with this software the 3D terrain model first was converted to a $3 \mathrm{D}$ elevation model of the ground level after which 3D roof lines of the buildings were added manually.

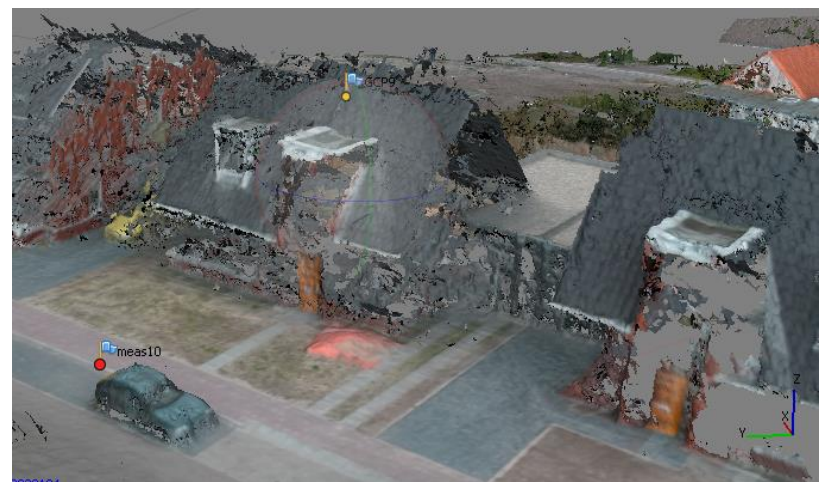

Figure 12: Artefacts in the 3D correlation process.

Measurement of coordinates in the derived model can be done in different ways. The point of interest can be measured in two or more of the original photos, after which the photo coordinates are transformed to 3D terrain coordinates. Or terrain coordinates can be directly measurement in the orthomosaic. Another option is to measure $3 \mathrm{D}$ coordinates in the stereo model, which is supported by Orbit GIS.

The obtained accuracy was especially evaluated for the second and third experiment of the Nunspeet area. With the PhotoScan 
Professional the model was created using 7 ground control points with an avarage total error of $3.1 \mathrm{~cm}$ and a standard deviation of $1.4 \mathrm{~cm}$. For 15 check points of parcel borders the coordinates were measured in the photos of the model. The found average planimetric error was $4.9 \mathrm{~cm}$ with a standard deviation of $2.3 \mathrm{~cm}$. The coordinates were also measured in the ortho-mosaic. For the control points this gave an average planimetric error of $3.5 \mathrm{~cm}$ with a standard deviation of $1.6 \mathrm{~cm}$, so almost the same as found in the 3D model. For the 15 check points an average planimetric error of $7.4 \mathrm{~cm}$ with a standard deviation of $6.3 \mathrm{~cm}$ was found, so here the effect of the artefacts in the ortho-mosaic are visible.

The results obtained by Orbit GIS give were even better, for the ground control points a standard deviation of 1 to $2 \mathrm{~cm}$ was found and for the measurements in the ortho-mosaic errors in the order of 2 to $3 \mathrm{~cm}$ were obtained. See Figure-13

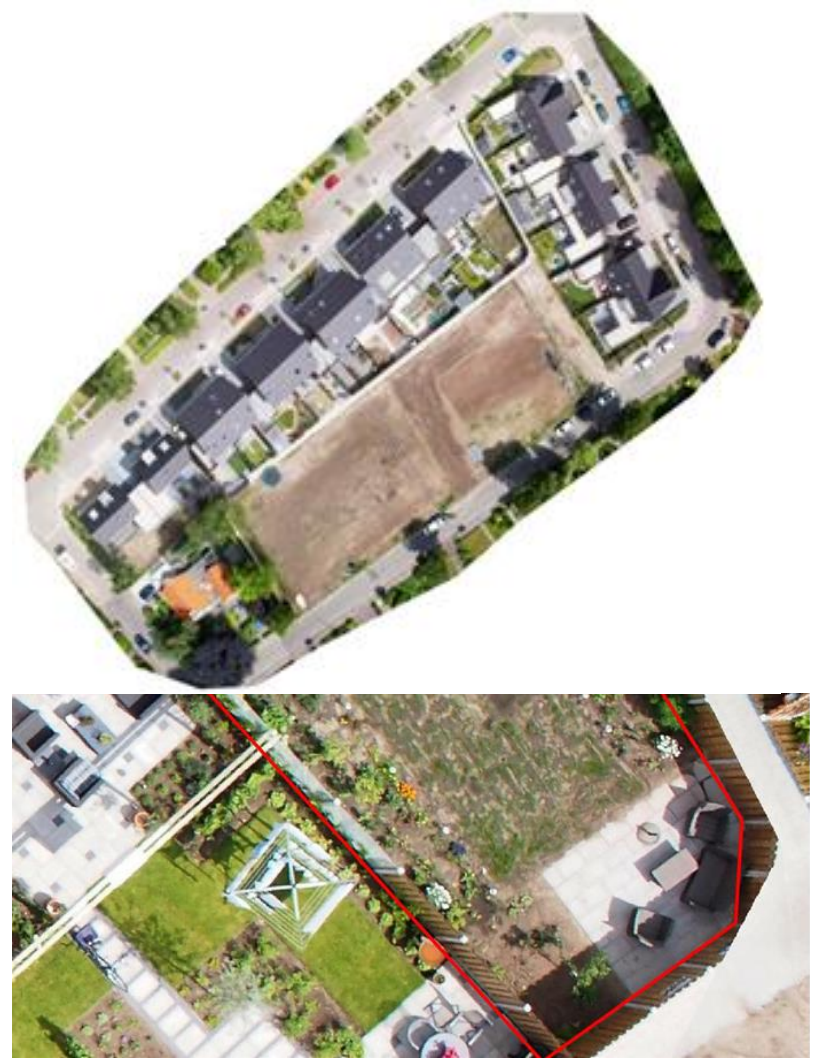

Figure 13: Orthophoto Nunspeet and zoomed-in orthophoto with cadastral borders (in red).

\section{DISCUSSION}

The finally obtained result of an ortho-photo with an average maximum geometrical precision of 3 centimetres is very useful. A cadastral employer can sketch cadastral borders and customers are able to give their interpretation about it. The average geometrical precision of three centimetres proves that the pictures have the same or better quality as conventional terrestrial surveying methods.

From the experiments it became clear that the accuracy of the measurements is influenced by multiple factors, including the quality of the camera, the camera calibration, the number and location of ground control points and the processing software used. During the three experiments the geometrical accuracy was extended from the first flight in Austerlitz to the last one in Nunspeet. In Austerlitz, camera parameters were not calibrated at all, which resulted in a geometrical inaccuracy which is in most cases more than $10 \mathrm{~cm}$. The last experiment in Nunspeet resulted in an average geometrical accuracy of 3 $\mathrm{cm}$ maximum.

Multiple SFM based photogrammetric software solutions are available, ranging from web services, standalone software packages, open software, to modules of professional photogrammetry/GIS suites. The functionality, type of products, user friendliness, accuracy and speed of these packages differs. It is depending on the work environment and objectives of the organisation what will be the optimal solution.

From an operational point of view a number of issues need to be solved. First, how to deal with ground control? This is an additional activity apart from the UAS fights. Probably it would be optimal to use natural points that are identifiable in the photos and that can be measured afterwards for multiple flights.

Second, how will customers react to these photos and how should they give their corrections or validations about it? And finally what are the juridical consequences of using these ortho-photos in the cadastral verification of borders? It is recommended to answer these questions in further research.

Next to the ortho-photo, some additional products can be generated. From the point clouds accurate terrain or elevation models can be computed. Secondly 3D texturized models can be generated, especially when manually measured rooflines are added. Thirdly stereoscopic visualisations can be generated and combined visualisations with other GIS layers are easily possible. These products might be valuable for other cadastral applications. Examples are areas which are poorly accessible or intensive infrastructural works (eg. Betuwelijn, Hanzenlijn). It is recommended to do some experiments in these cases as well.

\section{CONCLUSIONS AND RECOMMENDATIONS}

All experiments provide answers to the research question how useful an Unmanned Aerial System is in the juridical verification process of cadastral ownership at Kadaster. This results in the following conclusions:

- An UAS is a useful system for making high resolution photos above areas with multiple new parcel borders.

- The ortho-mosaics generated from the high resolution UAS photos are at least as accurate as conventional terrestrial surveying methods. Experiment showed geometrical accuracies with a maximum of 3 centimetres. This makes ortho-photos useful for the verification and measurement of ownership.

- Structure From Motion based software packages are well suited for accurate geometric processing of UAS based photos.

- High resolution UAS based photos / ortho-mosaics could be used also for other cadastral applications. Examples are areas which are poorly accessible or intensive infrastructural works. 
All conclusions lead to these recommendations:

- Start research to the juridical consequences of using orthophotos made by UAS in the juridical verification of borders of ownership.

- Define a pilot and start using ortho-photos made by UAS in a real cadastral surveying project and validate the results as first step for implementation in the juridical verification process;

- Start more research to the use of additional products of UAS-data, like the 3D height models, stereo representation or 3D realistic models.

- Define other possible experiments to use high resolution pictures taken by UAS for other cadastral applications, like infrastructural works or areas which are poorly accessible.

- Define a business case of the use of an UAS in the verification of borders of ownership.

\section{REFERENCES}

AscTec Falcon 8, http://www.asctec.de/uav-applications/aerialimaging/asctec-falcon-8/asctec-falcon-8/, May 2013

Cunningham, K., et al, 2011, Cadastral audit and assessment using unmanned aerial systems, International Archives of the Photogrammetry, Remote Sensing and Spatial Information Sciences, Vol. XXXVIII-1/C22

UAV-g 2011, Conference on Unmanned Aerial Vehicle in Geomatics

Eissenbeiss, H. (2009), “UAV Photogrammetry”, ETH Zurich; Flury, G. (2011), "Evaluering der Gleisleitungsvermessung mittels UAV”, ETH Zurich;

Handleiding Technische Werkzaamheden (HTW) Kadaster, Kadaster Apeldoorn, 1994;

Manyoky, M., et.al, 2011, Unmanned Aerial Vehicle in cadastral applications, International Archives of the Photogrammetry, Remote Sensing and Spatial Information Sciences, Vol. XXXVIII-1/C22

UAV-g 2011, Conference on Unmanned Aerial Vehicle in Geomatics

Microdrone MD4-1000,

http://www.microdrones.com/products/md4-1000/md4-1000key-information.php, May 2013

Neuvel, J.M.M. et.al., 2009, From Spatial Data to Synchronised Actions: The Network-centric Organisation of Spatial Decision Support for Risk and Emergency Management, Appl. Spatial Analysis, Springerlink.com

Ober, C. and Schindler, Prof. K. C., (2010), "Untersuchungen zum videomodus der Camera auf dem Octokopter", masterarbeit, ETH Zurich, Switzerland;

Orbit Strabo, http://www.orbitgis.com/store/product/orbitstrabo-photogrammetry, May 2013

PhotoModeler, 2013, PhotoModeler product description, http://www.photomodeler.com/products/modeler/default.html, May 2013
PhotoScan Professional, http://www.agisoft.ru/products/photoscan/professional/, May 2013

Smith, Gary S. "Digital Orthography and GIS", ESRI Conference 2011

VisualSFM, http://www.cs.washington.edu/homes/ccwu/vsfm/, May 2013 